\title{
Disease Response
}

National Cancer Institute

\section{Source}

National Cancer Institute. Disease Response. NCI Thesaurus. Code C50995.

The pathologic and/or clinical changes that result from treatment. The changes may

include eradication of detectable disease, stabilization of disease, or disease progression. 